\title{
Around the nonlinear Ryll-Nardzewski theorem
}

\author{
Andrzej Wiśnicki ${ }^{1}$
}

Received: 2 May 2019 / Revised: 18 November 2019 / Published online: 30 March 2020

(C) The Author(s) 2020

\section{Abstract}

Suppose that $Q$ is a weak* compact convex subset of a dual Banach space with the Radon-Nikodým property. We show that if $(S, Q)$ is a nonexpansive and norm-distal dynamical system, then there is a fixed point of $S$ in $Q$ and the set of fixed points is a nonexpansive retract of $Q$. As a consequence we obtain a nonlinear extension of the Bader-Gelander-Monod theorem concerning isometries in $L$-embedded Banach spaces. A similar statement is proved for weakly compact convex subsets of a locally convex space, thus giving the nonlinear counterpart of the Ryll-Nardzewski theorem.

Mathematics Subject Classification Primary 37B05; Secondary 28D05 • 47H10 . $54 \mathrm{H} 20$

\section{Introduction}

Fixed point theorems for groups and semigroups of mappings provide a powerful tool in diverse branches of mathematics. Kakutani-type theorems have found numerous applications in functional analysis, harmonic analysis and ergodic theory. Furstenberg's structure theorem and its consequences are fundamental tools in topological dynamics. The Bruhat-Tits theorem concerning complete metric spaces satisfying the parallelogram law turns out to be useful in differential geometry. Kazhdan's property (T) plays a prominent role in geometric group theory and related fields. Also, the recent Bader-Gelander-Monod theorem for affine isometries preserving a bounded set in $L$-embedded Banach spaces has several applications in group theory and in the theory of operator algebras, including the optimal solution to the old "derivation problem" in $L^{1}(G)$.

Dedicated to Professor Kazimierz Goebel on the occasion of his 80th birthday.

Communicated by Andreas Thom.

$凶$ Andrzej Wiśnicki

andrzej.wisnicki@up.krakow.pl

1 Department of Mathematics, Pedagogical University of Krakow, 30-084 Cracow, Poland 
One of the first general fixed point theorems for isometries, next to Kakutani-type theorems, was shown by Brodskiı̌ and Mil'man in [6] -all surjective isometries acting on a weakly compact, convex subset of a Banach space with normal structure have a common fixed point. In particular, a group of isometries of a uniformly convex or a uniformly smooth Banach space with bounded orbits has a fixed point. Since surjective isometries acting on the whole space are always affine, the last result holds true in all reflexive spaces as a consequence of the Ryll-Nardzewski theorem, and still more generally, in all duals of Asplund spaces, thus in all separable dual spaces provided the isometries are weak* continuous. But the Ryll-Nardzewski theorem goes beyond isometries and concerns a significant generalization of isometric dynamical systems (as defined after Theorem 1.1) called distal systems. Let us recall its classical version (see [24,25]).

Theorem 1.1 (Ryll-Nardzewski) Let $Q$ be a nonempty weakly compact convex subset of a locally convex space $(X, \tau)$ and let $(S, Q)$ be an affine and $\tau$-distal dynamical system. Then there is a fixed point of $S$ in $Q$.

By a dynamical system we mean a pair $(S, Q)$, where $S$ is a semigroup, $Q$ a topological space and there is a semigroup action $S \times Q \rightarrow Q$ of $S$ on $Q$ such that the mappings $Q \ni x \rightarrow s x \in Q$ are continuous (in the topology of $Q$ ) for each $s \in S$. If $X$ is a locally convex space whose topology $\tau$ is determined by a family $\mathcal{N}$ of seminorms on $X$ and $Q \subset X$, a dynamical system $(S, Q)$ is said to be isometric if $p(s x-s y)=p(x-y)$ for every $p \in \mathcal{N}, s \in S$ and $x, y \in Q$. A dynamical system is affine if $Q$ is convex and $s(\alpha x+(1-\alpha) y)=\alpha s x+(1-\alpha) s y$ for every $\alpha \in[0,1], s \in S$ and $x, y \in Q$. A dynamical system is compact if $Q$ is compact, and $\tau$-distal (or $\tau$-noncontracting) if

$$
0 \notin \overline{\{s x-s y: s \in S\}}^{\tau}
$$

for every pair of distinct points $x, y \in Q$.

Ryll-Nardzewski's theorem involves the interplay between the strong and weak topology, and considerably improves Hahn's and Kakutani's fixed point theorems. In particular, since isometric systems are distal and $S$ is a semigroup, it follows that all continuous affine isometries, not necessarily surjections, defined on a weakly compact convex set $Q$ have a common fixed point. The question naturally arises as to whether there is a nonlinear counterpart of this result or, more generally, whether there exists a nonlinear version of Ryll-Nardzewski's theorem.

Two remarks are in order. Note first that Alspach's example [1] shows that there is a fixed-point free isometry on a weakly compact convex subset of $L_{1}[0,1]$. Thus, the assumption that the mappings $Q \ni x \rightarrow s x \in Q$ are weakly continuous cannot be relaxed in general. (Another choice is to put some additional requirements on $Q$ but it is another interesting story). Secondly, Boyce [5] showed an example of two commuting continuous maps of [0,1] into itself without a common fixed point, and Huneke [15] showed another such example with Lipschitzian mappings. However, DeMarr [8] was able to prove that a commutative family of nonexpansive (i.e., 1-Lipschitz) mappings defined on a convex compact subset of a Banach space has a common fixed point. Thus a natural requirement in the nonlinear case is the nonexpansivity of a dynamical system 
and the program of studying fixed point properties of semigroups of nonexpansive mappings has already been performed in the context of amenable semigroups (see [18] and references therein).

In this paper we show the nonlinear version of Ryll-Nardzewski's theorem and some of its generalizations (see [12] for a thorough study of this problem). If $Q$ is a subset of a locally convex space whose topology $\tau$ is determined by a family $\mathcal{N}$ of seminorms, a dynamical system $(S, Q)$ is said to be $\mathcal{N}$-nonexpansive (or briefly, nonexpansive if $\mathcal{N}$ is fixed) if $p(s x-s y) \leq p(x-y)$ for every $p \in \mathcal{N}, s \in S$ and $x, y \in Q$. Here is a summary of our main results.

Theorem A Let $Q$ be a weak* compact convex subset with the Radon-Nikodým property of a dual Banach space and let $(S, Q)$ be a nonexpansive and norm-distal dynamical system. Then there is a fixed point of $S$ in $Q$. Moreover, the set of fixed points is a nonexpansive retract of $Q$.

In particular, Theorem $\mathrm{A}$ is valid if $Q$ is a weakly compact convex subset of any Banach space or a norm-separable weak* compact convex subset of a dual Banach space. Furthermore, if $Q$ is a weak* compact convex subset of the dual of an Asplund space, we obtain the nonlinear version of Namioka-Phelps' theorem (see [23, Theorem 15]). In locally convex spaces, we have the following counterpart of the Ryll-Nardzewski Theorem.

Theorem B Let $Q$ be a nonempty weakly compact convex subset of a locally convex space $(X, \tau)$ and let $(S, Q)$ be a nonexpansive and $\tau$-distal dynamical system. Then there is a fixed point of $S$ in $Q$. Moreover, the set of fixed points is a nonexpansive retract of $Q$.

The qualitative part of both Theorems A and B is a consequence of Bruck's theorem [7, Theorem 3]. Next, we have the following nonlinear extension of the BaderGelander-Monod theorem [2, Theorem A].

Theorem C Let X be an L-embedded Banach space and let $(S,(X$, weak)) be a nonexpansive and norm-distal dynamical system. If there is a bounded set $A \subset X$ such that $s(A)=A$ for all $s \in S$, then there is a fixed point of $S$ located in the Chebyshev center of $A$.

Theorem $\mathrm{C}$ follows from the more general Theorem 5.3 in Sect. 5. In particular, it holds for a semigroup of weakly continuous isometries preserving $A$. Note that in [2] a similar statement is asserted for a group of affine isometries.

The organization of the paper is as follows. In Sect. 2 we collect the basic tools that we shall use throughout the proofs, including the fundamental for our purpose Furstenberg's fixed point theorem. In Sect. 3 we show a general nonlinear Ryll-Nardzewski type theorem in Banach spaces and its consequences. Section 4 presents the proof of the first part of Theorem B. In Sect. 5 we apply the previous results to prove the qualitative parts of Theorems A, B and an extension of Theorem C to dynamical systems in $L$-embedded sets. The paper is concluded with a nonlinear generalization of Fan's theorem [9, Theorem 1] concerning the orbits of semigroups of linear contractions in the case of reflexive Banach spaces. 


\section{Main tools}

In this section we list the main components that we shall use to prove the nonlinear Ryll-Nardzewski theorem and its generalizations.

A bounded subset $A$ of a Banach space $X$ is called dentable if for each $\varepsilon>0$ there is $x \in A$ such that $x \notin \overline{\mathrm{co}}(A \backslash B(x, \varepsilon))$. We recall the following characterization of a set with the Radon-Nikodým property (RNP for short): a bounded closed convex set $C \subset X$ has the RNP iff every bounded nonempty subset of $C$ is dentable. A Banach space $X$ is said to have the RNP if its unit ball has the RNP. It is well known that any weakly compact convex subset of a Banach space has the RNP as well as any norm-separable weak* compact convex subset of a dual space. Moreover, a Banach space $X$ is Asplund iff $X^{*}$ has the RNP.

The notion of dentability is closely related with the concept of fragmentability, invented by Jayne and Rogers [16], that is crucial for the geometric approach to RyllNardzewski's theorem and its generalizations. The related ideas go back to the works of Glasner [11], Hansel and Troallic [13], Namioka and Phelps [23], Veech [26], and culminate in a very general Lemma 1.2 of [12] allowing one to lift the $\tau$-distality of $(S, Q)$ to the original, usually weaker topology. Let $(X, \omega)$ be a topological space and let $\rho$ be a metric on $X$. We say that $(X, \omega)$ is $\rho$-fragmented if for every $\varepsilon>0$ and a nonempty set $A \subset X$ there is an $\omega$-open set $U$ in $X$ such that $U \cap A \neq \emptyset$ and $\rho$ $\operatorname{diam}(U \cap A)<\varepsilon$. A rather straightforward application of the Baire category theorem shows that a compact space $(X, \omega)$ is $\rho$-fragmented iff for each $\omega$-closed subset $A$ of $X$, the identity map id $:(A, \omega) \rightarrow(A, \rho)$ has a point of continuity. Thus every weak* compact convex set with the RNP is norm-fragmented (see, e.g., [4, Theorem 4.2.13]).

The fundamental tool for our results is the following consequence of Furstenberg's structure theorem [10], extended from metrizable to arbitrary compact affine systems by Namioka (see [21, Theorem 4.1], [22, Theorem 4.1]).

Theorem 2.1 (Furstenberg's fixed point theorem) Let $(S, Q)$ be a compact affine dynamical system. Suppose there exists a nonempty compact $S$-invariant subset $K$ (i.e., $s(K) \subset K$ for each $s \in S$ ) of $Q$ such that $(S, K)$ is distal. Then there is a common fixed point of $S$ in $Q$.

It follows from Theorem 2.1 that every distal compact dynamical system admits an invariant Radon probability measure. The Radon-Nikodym property implies that every Radon measure on a weak* compact convex set with the RNP has norm-separable support (see, e.g., [4, Theorem 4.3.11]).

The next component is the following observation of DeMarr [8, Lemma 1] that also follows from the characterization of normal structure in [6]. Recall that a point $x$ of a bounded set $A \subset X$ is called diametral if $\sup _{y \in A}\|x-y\|=\operatorname{diam} A$. A convex set $K \subset X$ is said to have normal structure if each bounded, convex subset $A$ of $K$ with $\operatorname{diam} A>0$ contains a nondiametral point.

Lemma 2.2 Let $X$ be a Banach space and let $K$ be a nonempty compact subset of $X$. Then there exists $u \in \overline{\mathrm{co}} K$ such that $\sup \{\|x-u\|: x \in K\}<\operatorname{diam} K$ provided $\operatorname{diam} K>0$. 
It follows that every compact convex subset of $X$ has normal structure. Note that the result remains unchanged if we replace $X$ by any locally convex space and a norm by a continuous seminorm.

The link between the weak, weak* and norm compactness is given by the method developed in the nonlinear case by Hsu, To-Ming Lau and Takahashi (see [14], [17, Lemma 5.2]), and Bartoszek [3, Lemma 1]. We reformulate the result of Hsu in the case of locally convex spaces. Let $X$ be a locally convex space whose topology $\tau$ is determined by a family $\mathcal{N}$ of seminorms on $X$ and let $K \subset X$. We say that $K$ is a minimal weakly compact $S$-invariant subset of $X$ if there is no proper (nonempty) weakly compact $S$-invariant set $K_{0} \subsetneq K$.

Lemma 2.3 Let $(S, K)$ be an $\mathcal{N}$-nonexpansive dynamical system, where $K$ is a minimal weakly compact $S$-invariant and $\tau$-separable subset of a locally convex space $(X, \tau)$ such that $s(K)=K$ for each $s \in S$. Then $K$ is $\tau$-totally bounded.

Proof Let $U=\left\{x \in X: p_{1}(x)<\varepsilon, \ldots, p_{n}(x)<\varepsilon\right\}, p_{1}, \ldots, p_{n} \in \mathcal{N}, \varepsilon>0$, be a $\tau$-open neighbourhood of 0 and take a convex $\tau$-closed neighbourhood $V$ of 0 such that $V-V \subset U$. Since $K$ is $\tau$-separable, there exists a sequence $\left\{x_{n}\right\} \subset K$ such that $K \subset \bigcup_{n=1}^{\infty}\left(x_{n}+V\right)$. Since $(K$, weak) is a Baire space and each translate of $V$ is weakly closed, there is a weakly open neighbourhood $W$ of 0 and $z, x_{i} \in K$ such that

$$
(z+W) \cap K \subset\left(x_{i}+V\right) \cap K \subset(z+U) \cap K .
$$

Take a weakly open neighbourhood $W^{\prime}$ of 0 such that $W^{\prime}+W^{\prime} \subset W$ and a $\tau$-open neighbourhood of $0, U^{\prime}=\left\{x \in X: q_{1}(x)<\varepsilon^{\prime}, \ldots, q_{m}(x)<\varepsilon^{\prime}\right\}, q_{1}, \ldots, q_{m} \in$ $\mathcal{N}, \varepsilon^{\prime}>0$, such that $U^{\prime} \subset W^{\prime}$. By $\tau$-separability, there is a sequence $\left\{y_{n}\right\} \subset K$ such that $K \subset \bigcup_{n=1}^{\infty}\left(y_{n}+U^{\prime}\right)$. Since $K$ is minimal $S$-invariant, $\{s y: s \in S\}$ is weakly dense in $K$ for each $y \in K$, and hence we can choose by induction a sequence $\left\{s_{n}\right\} \subset S$ such that $s_{1} y_{1}, s_{2} s_{1} y_{2}, \ldots, s_{n} s_{n-1} \ldots, s_{1} y_{n}, \ldots \in z+W^{\prime}$. It follows from $\mathcal{N}$-nonexpansivity that

$$
s_{n} s_{n-1} \ldots s_{1}\left(\left(y_{n}+U^{\prime}\right) \cap K\right) \subset s_{n} s_{n-1} \ldots s_{1} y_{n}+U^{\prime} \subset z+W
$$

for each $n$, and thus $K \subset \bigcup_{n=1}^{\infty}\left(s_{n} s_{n-1} \ldots s_{1}\right)^{-1}(z+W)$. Since $K$ is weakly compact, there exists a finite subcover $K \subset \bigcup_{n=1}^{p}\left(s_{n} s_{n-1} \ldots s_{1}\right)^{-1}(z+W)$. Now we have

$$
\begin{aligned}
K & =s_{p} s_{p-1} \ldots s_{1}(K) \subset \bigcup_{n=2}^{p} s_{p} s_{p-1} \ldots s_{n}((z+W) \cap K) \cup((z+W) \cap K) \\
& \subset \bigcup_{n=2}^{p} s_{p} s_{p-1} \ldots s_{n}((z+U) \cap K) \cup((z+U) \cap K)
\end{aligned}
$$

and from the nonexpansivity, $K \subset \bigcup_{n=2}^{p}\left(s_{p} s_{p-1} \ldots s_{n} z+U\right) \cup(z+U)$, that is, $K$ is totally bounded. 
Note that in Banach spaces the above argument works when $K$ is weak* compact too (see [17, Lemma 5.2]). Another approach to this problem, adapted in Sect. 3, was given by Bartoszek [3, Lemma 1].

In what follows, we show how the results described in this section interact with one another and with some classical arguments in this field to prove nonlinear RyllNardzewski type theorems.

\section{Fixed points of distal systems and the Radon-Nikodým property}

In this section we prove a general nonlinear fixed point theorem of Ryll-Nardzewski type in Banach spaces.

Theorem 3.1 Let $Q$ be a weak* compact convex subset with the Radon-Nikodym property of a dual Banach space $X$ and let $(S, Q)$ be a nonexpansive and norm-distal dynamical system. Then there is a fixed point of $S$ in $Q$.

Proof By Kuratowski-Zorn's lemma, we can assume without loss of generality that $Q$ is a minimal $S$-invariant weak* compact convex subset with the RNP of $X$. Let $K$ be a minimal $S$-invariant weak* compact subset of $Q$. We first prove that $(S, K)$ is weak*-distal. A trick is to 'lift' the distality, using fragmentability. A general result of this type, inspired by [13, Proposition 2], is shown in [12, Lemma 1.2]. We present this argument in the case of norm and weak* topologies. Fix $x, y \in K$. By norm-distality, there is $\varepsilon>0$ such that

$$
\|s x-s y\|>\varepsilon
$$

for every $s \in S$. Suppose conversely that $S$ is not weak ${ }^{*}$-distal on $K$, i.e., there are nets $\left(s_{\alpha} x\right),\left(s_{\alpha} y\right)$ such that $w^{*}-\lim s_{\alpha} x=w^{*}-\lim s_{\alpha} y=u$ for some $u \in K$. Notice

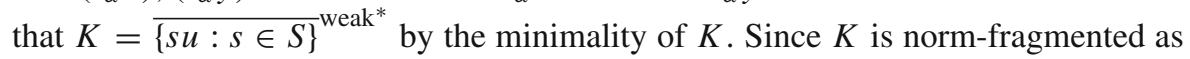
a subset of a weak* compact convex set $Q$ with the RNP, there is a weak*-open set $U$ in $Q$ such that $U \cap K \neq \emptyset$ and $\operatorname{diam}(U \cap K)<\varepsilon$. Hence there exists $s_{0} \in S$ such that $p=s_{0} u \in U \cap K$. Then

$$
w^{*}-\lim s_{0} s_{\alpha} x=p=w^{*}-\lim s_{0} s_{\alpha} y
$$

and thus $s_{0} s_{\alpha} x, s_{0} s_{\alpha} y \in U \cap K$ eventually. But this contradicts (3.1) and $S$ is weak*distal on $K$.

Let $C(K)$ denote the space of continuous functions on $K$ and define $(f \cdot s)(t)=$ $f(s t)$ for $f \in C(K)$ and $s, t \in S$. Let $T_{s} f=f \cdot s$ for each $s \in S$. Notice that $(S, P(K))$ is an affine dynamical system with the action $s \cdot \mu=T_{s}^{*}(\mu)$, where $P(K)$ is the convex weak* ${ }^{*}$-compact set of all means on $C(K)$ (i.e., Radon probability measures on $K$ with the weak ${ }^{*}$ topology) and $T_{s}^{*}: C(K)^{*} \rightarrow C(K)^{*}$ is the adjoint of $T_{s}$. Let $\phi: K \rightarrow P(K)$ be the natural embedding of $K$ into $P(K)$ defined by $\phi(x)(f)=f(x)$. Then $\phi$ is an isomorphism of systems $(S, K)$ and $(S, \phi(K))$. Thus $(S, \phi(K))$ is weak* distal since $(S, K)$ is and, by Theorem 2.1 , there is a fixed point 
$\mu$ of $S$ in $P(K)$, that is, $\mu$ is an $S$-invariant Radon probability measure on $K$ (with respect to weak* topology).

Define $K_{0}=\operatorname{supp}(\mu)$ and notice that $\mu\left(s^{-1}\left(K_{0}\right)\right)=\mu\left(K_{0}\right)=1$. Furthermore, $s^{-1}\left(K_{0}\right)$ is weak* closed and $K_{0}$ is the least weak* closed subset of $K$ of full measure. Hence $K_{0} \subset s^{-1}\left(K_{0}\right)$. Similarly,

$$
\mu\left(s\left(K_{0}\right)\right)=\mu\left(s^{-1}\left(s\left(K_{0}\right)\right)\right)=\mu\left(K_{0}\right)=1
$$

and consequently, from weak* compactness of $s\left(K_{0}\right), K_{0} \subset s\left(K_{0}\right)$. Thus $s\left(K_{0}\right)=K_{0}$ for every $s \in S$ and, since $K$ is a minimal $S$-invariant weak* compact subset of $Q$, $K=K_{0}$. We show that $K$ is norm-compact. Since $K$ is weak* compact and $Q$ has the RNP, the identity map id $:\left(K\right.$, weak $\left.^{*}\right) \rightarrow(K$, norm $)$ has a point of continuity $x$ (see, e.g., [4, Theorem 4.2.13]). It follows that for every $\varepsilon>0$ there is a weak ${ }^{*}$ open neighbourhood $U$ of $x$ such that $\|x-y\|<\varepsilon$ for each $y \in U \cap K$. But $x \in \operatorname{supp}(\mu)$ and hence

$$
\mu(\{y \in K:\|x-y\|<\varepsilon\}) \geq \mu(U \cap K)>0
$$

for each $\varepsilon>0$.

Now we follow partly the argument of Bartoszek [3, Lemma 1]. Fix $\varepsilon>0$ and let $\mu(\{y \in K:\|x-y\|<\varepsilon\})=\delta>0$. Notice that by nonexpansivity,

$$
\{y \in K:\|x-y\|<\varepsilon\} \subset s^{-1}(\{y \in K:\|s x-y\|<\varepsilon\})
$$

for each $s \in S$ and, since $\mu$ is $S$-invariant,

$$
\mu(\{y \in K:\|s x-y\|<\varepsilon\}) \geq \mu(\{y \in K:\|x-y\|<\varepsilon\})=\delta .
$$

It follows that a number of elements $s_{1}, s_{2}, \ldots, s_{k}$ such that

$$
\left\|s_{i} x-s_{j} x\right\| \geq 2 \varepsilon
$$

for each $1 \leq i \neq j \leq k$ is bounded by $1 / \delta$. Hence $\{s x: s \in S\}$ is norm-totally

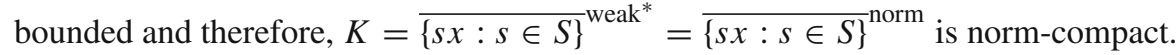

We show that $K$ consists of a single point. Suppose that $r=\operatorname{diam} K>0$. Then by Lemma 2.2, there is $u \in \overline{\mathrm{co}} K$ such that $r_{0}=\sup \{\|u-y\|: y \in K\}<r$. Define

$$
Q_{0}=\left\{x \in Q:\|x-y\| \leq r_{0} \text { for all } y \in K\right\} .
$$

Then $u \in Q_{0}$ and $Q_{0}$ is a weak* compact convex proper subset of $Q$. Since the system $(S, Q)$ is nonexpansive and $s(K)=K$, it follows that $s\left(Q_{0}\right) \subset Q_{0}$ for each $s \in S$ which contradicts the minimality of $Q$. Thus diam $K=0$ and $K$ consists of a single point which is a common fixed point of $S$ in $K$.

As a consequence, we obtain the nonlinear version of Namioka-Phelps' theorem [23, Theorem 15]. Recall that a Banach space $X$ is Asplund if every continuous convex 
function on any open convex subset $U$ of $X$ is Fréchet differentiable on a dense $G_{\delta^{-}}$ subset of $U$. By the results of Namioka, Phelps and Stegall, $X$ is Asplund iff $X^{*}$ has the RNP.

Corollary 3.2 Suppose $X$ is an Asplund space and $Q$ a weak ${ }^{*}$ compact convex subset of $X^{*}$. If $(S, Q)$ is a nonexpansive and norm-distal dynamical system, then there is a fixed point of $S$ in $Q$.

Similarly, since every separable weak* compact convex subset of a dual space has the RNP, we have

Corollary 3.3 Let $Q$ be a separable weak* compact convex subset of a dual Banach space $X^{*}$ and let $(S, Q)$ be a nonexpansive and norm-distal dynamical system. Then there is a fixed point of $S$ in $Q$.

Furthermore, every weakly compact convex subset of a Banach space $X$ can be regarded as a weak* compact convex subset (with the RNP) of $X^{* *}$. Hence we obtain the nonlinear Ryll-Nardzewski theorem in a Banach space.

Corollary 3.4 Let $Q$ be a weakly compact convex subset of a Banach space $X$ and let $(S, Q)$ be a nonexpansive and norm-distal dynamical system. Then there is a fixed point of $S$ in $Q$.

In the next section we generalize Corollary 3.4 to locally convex spaces.

\section{Nonlinear Ryll-Nardzewski's theorem in locally convex spaces}

In a locally convex space $(X, \tau)$, the arguments in the proof of Theorem 3.1 are not completely applicable. However, in the case of weakly compact convex sets, we can reduce the problem (by a classical argument) to $S$ being countable and then use Lemma 2.3.

Theorem 4.1 Let $Q$ be a weakly compact convex subset of a locally convex space $(X, \tau)$ whose topology $\tau$ is determined by a family $\mathcal{N}$ of seminorms on $X$. If a dynamical system $(S, Q)$ is $\mathcal{N}$-nonexpansive and $\tau$-distal, then there is a fixed point of $S$ in $Q$.

Proof By weak compactness, it is sufficient to show that each finite subset of $S$ has a common fixed point in $Q$. Hence we can assume that $S$ is countable and $Q$ is a minimal $S$-invariant weakly compact convex subset of $X$. Let $K$ be a minimal $S$ invariant weakly compact subset of $Q$.

We show that $S$ is weakly-distal on $K$. If not, then there are $x, y, u \in K$ and nets $\left(s_{\alpha} x\right),\left(s_{\alpha} y\right)$ such that $w$-lim $s_{\alpha} x=w$ - $\lim s_{\alpha} y=u$. Notice that $K=\overline{\{s u: s \in S\}}^{\text {weak }}$ by the minimality of $K$. Since $(S, K)$ is $\tau$-distal, there is a $\tau$-neighborhood $U$ of 0 such that $s(x)-s(y) \notin U$ for each $s \in S$. Let $V$ be a convex $\tau$-closed neighborhood of 0 such that $V-V \subset U$. By Mazur's lemma, $K \subset \overline{c o}^{\tau}\{s u: s \in S\}$ and, since $S$ is countable, $K$ is $\tau$-separable. Hence there exists a sequence $\left\{y_{n}\right\} \subset K$ such 
that $K \subset \bigcup_{n=1}^{\infty}\left(y_{n}+V\right)$. From Baire's category theorem, there is a weakly open neighbourhood $W$ of 0 and $z, y_{i} \in K$ such that $(z+W) \cap K \subset y_{i}+V$. Thus there is $s_{0} \in S$ such that $s_{0} u \in z+W$. It follows that

$$
w-\lim s_{0} s_{\alpha} x=s_{0} u=w-\lim s_{0} s_{\alpha} y
$$

and eventually $s_{0} s_{\alpha} x, s_{0} s_{\alpha} y \in(z+W) \cap K \subset y_{i}+V$. Hence $s_{0} s_{\alpha} x-s_{0} s_{\alpha} y$ is eventually in $V-V \subset U$ but it contradicts our choice of $U$. Thus $S$ is weakly-distal on $K$.

Now, as in the proof of Theorem 3.1, we can show that $K$ admits an $S$-invariant Radon probability measure and $s(K)=K$ for each $s \in S$. Moreover, $K$ is $\tau$-separable and it follows from Lemma 2.3 that $K$ is totally bounded. We can certainly assume that $(X, \tau)$ is complete (since otherwise we consider the closure of $K$ in the completion of $X$ ). Thus $K$ is $\tau$-compact. If $K$ consists of more than one point, then there exists a seminorm $q \in \mathcal{N}$ such that $r=\sup \{q(x-y): x, y \in K\}>0$. Then by a counterpart of Lemma 2.2, there is $u \in \overline{\mathrm{co}} K$ such that $r_{0}=\sup \{q(u-y): y \in K\}<r$. Define

$$
Q_{0}=\left\{x \in Q: q(x-y) \leq r_{0} \text { for all } y \in K\right\} .
$$

Then $u \in Q_{0}$ and $Q_{0}$ is a weakly compact convex proper subset of $Q$. Since the action is $\mathcal{N}$-nonexpansive and $s(K)=K, s \in S$, we have $s\left(Q_{0}\right) \subset Q_{0}$ for each $s \in S$ which contradicts the minimality of $Q$. Thus $K$ consists of a single point $x$ and $s x=x$ for every $s \in S$.

There is a natural generalization of Asplund Banach spaces, introduced in [20, Definition 4.1] (see also [12, Definition 1.10]): a locally convex space $(X, \tau)$ is called a Namioka-Phelps space if every equicontinuous subset $K$ in $X^{*}$ is (weak ${ }^{*}, \xi^{*}$ )fragmented, where $\xi^{*}$ denotes the natural uniform structure of $X^{*}$. It is shown in [20] that the class of Namioka-Phelps spaces contains in particular Fréchet differentiable spaces, semireflexive spaces and nuclear spaces, and is closed under taking subspaces, products and direct sums (see also [12, Remark 1.12]). Since the weak compactness of $Q$ is applied in the proof of Theorem 4.1 in a substantial way to show the $\tau$-separability of $K$, it is not clear how to extend Corollary 3.2 to the case of locally convex spaces. This leads to the following natural questions.

Question 1 Is it true that Corollary 3.2 remains true for Namioka-Phelps spaces?

More generally, we can ask:

Question 2 Do there exist nonlinear counterparts of Theorems 1.5 and 1.6 in [12]?

\section{Applications}

In 2012, Bader, Gelander and Monod [2] gave a beautiful proof of a fixed point theorem in $L$-embedded Banach spaces. One of its consequences is the optimal solution to the following "derivation problem" : if $G$ is a locally compact group, then any derivation 
from the convolution algebra $L^{1}(G)$ to $M(G)$ is inner. The problem was studied since 1960s and proved for the first time by Losert [19, Theorem 1.1]. We apply Theorem 3.1 to show a nonlinear extension of Bader-Gelander-Monod's theorem [2, Theorem A].

Recall that a Banach space $X$ is said to be $L$-embedded if its bidual $X^{* *}$ can be decomposed as $X^{* *}=X \oplus_{1} X_{S}$ for some $X_{s} \subset X^{* *}$ (with the norm being the sum of norms of $X$ and $X_{S}$ ). The class of $L$-embedded Banach spaces includes all $L_{1}$ spaces, preduals of von Neumann algebras and the Hardy space $H_{1}$. We need the following generalization.

Definition 5.1 (see [18]) Let $C$ be a nonempty subset of a Banach space $X$ and denote by $\bar{C}^{\text {wk* }^{*}}$ the closure of $C$ in $X^{* *}$ in the weak* topology of $X^{* *}$. We say that $C$ is $L$-embedded if there is a subspace $X_{S}$ of $X^{* *}$ such that $X \oplus_{1} X_{S} \subset X^{* *}$ and $\bar{C}^{\mathrm{wk}} \subset C \oplus_{1} X_{S}$.

It was proved in [18] that every $L$-embedded set is weakly closed. Moreover, a Banach space is $L$-embedded iff its unit ball is $L$-embedded. Notice that a weakly compact subset $C$ of any Banach space $X$ is $L$-embedded since $\bar{C}^{\text {wk* }}=C$.

If $A, C$ are subsets of a Banach space $X$ with $A$ bounded, we define the Chebyshev radius of $A$ in $C$ by

$$
r_{C}(A)=\inf _{x \in C} \sup _{y \in A}\|x-y\|
$$

and the Chebyshev center of $A$ in $C$ by

$$
E_{C}(A)=\left\{x \in C: \sup _{y \in A}\|x-y\|=r_{C}(A)\right\} .
$$

Lemma 5.2 (see [18, Lemma 3.3]) Let $C$ be an L-embedded subset of a Banach space $X$ and $A$ a bounded subset of $X$. Then the Chebyshev center $E_{C}(A)$ is weakly compact.

Combining Corollary 3.4 with Lemma 5.2 yields the following extension of [2, Theorem A].

Theorem 5.3 Let $Q$ be a bounded convex L-embedded subset of a Banach space $X$ and let $(S,(Q$, weak $))$ be a nonexpansive and norm-distal dynamical system. If $Q$ contains a bounded subset $A$ such that $s(A)=A$ for all $s \in S$, then there is a fixed point of $S$ in $E_{Q}(A)$.

Proof Notice that $E_{Q}(A)$ is convex, $s\left(E_{Q}(A)\right) \subset E_{Q}(A)$ and by Lemma 5.2, $E_{Q}(A)$ is weakly compact. Therefore, we can apply Corollary 3.4 to obtain a fixed point of $S$ in $E_{Q}(A)$.

Corollary 5.4 Let A be a non-empty bounded subset of an L-embedded Banach space $X$. Then there is a point in $X$ fixed by every weakly continuous isometry of $X$ into $X$ preserving A. Moreover, one can choose a fixed point which minimizes $\sup _{a \in A}\|x-a\|$ over all $x \in X$. 
Note that in [2] a similar statement is asserted for affine isometries.

Our next result concerns the qualitative information about the structure of the set of fixed points of nonexpansive distal systems. We shall rely on the following consequence of Bruck's theorem [7, Theorem 3].

Theorem 5.5 Let $Q$ be a compact Hausdorff topological space and $S$ a (discrete) semigroup of mappings on $Q$. Suppose that $S$ is compact in the topology of pointwise convergence and each nonempty closed $S$-invariant subset of $Q$ contains a fixed point of $S$. Then there exists in $S$ a retraction of $Q$ onto $F(S)=\{x \in Q: s x=x$ for every $s \in S\}$.

Note that a retraction in the above theorem is simply a mapping $r: Q \rightarrow F(S)$ such that $r \circ r=r$. (The continuity of $r$ in the topology of $Q$ is not required). The following theorem is the qualitative part of Theorem A alluded to in the introduction.

Theorem 5.6 Let $Q$ be a weak* compact convex subset with the RNP of a dual Banach space $X$ and let $(S, Q)$ be a nonexpansive and norm-distal dynamical system. Then the set $F(S)$ of fixed points of $S$ is a nonexpansive retract of $Q$.

Proof Put $\hat{S}=\{T: Q \rightarrow Q \mid T$ is nonexpansive and $F(S) \subset F(T)\}$. Notice that $Q^{Q}$ is compact in the product topology when $Q$ is given the weak* topology and the product topology is the topology of weak* pointwise convergence. Moreover, $\hat{S} \subset Q^{Q}$ is closed in this topology since

$$
\left\|w^{*}-\lim T_{\alpha} x-w^{*}-\lim T_{\alpha} y\right\| \leq \liminf _{\alpha}\left\|T_{\alpha} x-T_{\alpha} y\right\| \leq\|x-y\|
$$

for every $x, y \in Q$ and every convergent net $\left\{T_{\alpha}\right\} \subset \hat{S}$, and $w^{*}-\lim T_{\alpha} x=x$ whenever $s x=x, s \in S$. Thus $\hat{S}$ is compact in the topology of weak ${ }^{*}$ pointwise convergence and $F(S)=F(\hat{S})$. Let $Q_{0}$ be a weak ${ }^{*}$ closed $\hat{S}$-invariant subset of $Q$. Choose $x \in Q_{0}$ and notice that $\hat{Q}=\{T x: T \in \hat{S}\}$ is a weak* compact $\hat{S}$-invariant subset of $Q_{0}$. Moreover, $\hat{Q}$ is convex since $\alpha T_{1}+(1-\alpha) T_{2} \in \hat{S}$ if $T_{1}, T_{2} \in \hat{S}$ and $\alpha \in[0,1]$. By Theorem 3.1, there is a fixed point of $\hat{S}$ in $\hat{Q} \subset Q_{0}$. Now it follows from Theorem 5.5 that there exists in $\hat{S}$ a retraction of $Q$ onto $F(\hat{S})=F(S)$. But every element in $\hat{S}$ is nonexpansive (though, not necessarily weak* continuous).

In a similar way we have the qualitative part of Theorem B.

Theorem 5.7 Let $Q$ be a weakly compact convex subset of a locally convex space $(X, \tau)$ and let $(S, Q)$ be an $\mathcal{N}$-nonexpansive and $\tau$-distal dynamical system. Then the set $F(S)$ of fixed points of $S$ is an $\mathcal{N}$-nonexpansive retract of $Q$.

We end the paper with the following nonlinear extension of Fan's result [9, Theorem $1]$ in reflexive spaces. Let $X$ be a Banach space and $E$ be a subset of $X^{*}$. We will say that a dynamical system $(S, E)$ is deflating if there exist distinct $\varphi_{1}, \varphi_{2} \in E$ such that for every absolutely convex, weakly compact set $C \subset X$ and any $\varepsilon>0$, there is $s \in S$ such that $\left|\left(s \varphi_{1}\right)(y)-\left(s \varphi_{2}\right)(y)\right|<\varepsilon$ for every $y \in C$. 
Theorem 5.8 Let $X$ be a reflexive Banach space, $z \in X,\|z\|=1$ and $Q=\{\varphi \in$ $\left.X^{*}:\|\varphi\|=\varphi(z)=1\right\} \subset E$. Let $(S,(Q$, weak $))$ be a non-deflating and normnonexpansive dynamical system. Then there exists $\psi \in Q$ such that $s \psi=\psi$ for all $s \in S$.

Proof It is clear that $Q$ is a convex weakly compact subset of $X^{*}$. Let $\varphi_{1}, \varphi_{2}$ be two distinct elements of $Q$. Since $S$ is non-deflating, there is a weakly compact absolutely convex subset $C$ of $X$ and $\varepsilon>0$ such that for every $S \in S$ there is $y_{0} \in C$ satisfying $\left|\left(s \varphi_{1}\right)\left(y_{0}\right)-\left(s \varphi_{2}\right)\left(y_{0}\right)\right| \geq \varepsilon$. Thus $\left\{\varphi \in X^{*}:|\varphi(y)|<\varepsilon\right.$ for $\left.y \in C\right\}$ is a $\tau\left(X^{*}, X\right)$ neighbourhood of 0 with respect to the Mackey topology $\tau\left(X^{*}, X\right)$ of $X^{*}$ which is disjoint from the set $\left\{s \varphi_{1}-s \varphi_{2}: s \in S\right\}$. Since $X$ is reflexive, $\tau\left(X^{*}, X\right)$ coincides with the norm topology and thus $(S, Q)$ is norm-distal. Now the thesis follows from Corollary 3.4 .

Let $z \in X,\|z\|=1$, and let $S$ be a semigroup of linear mappings $s: X \rightarrow X$ such that $\|u\| \leq 1$ and $s(z)=z$ for each $s \in S$. Suppose that $S$ has no direction of deflation, i.e., for every $0 \neq \varphi \in X^{*}$ there is an absolutely convex, weakly compact subset of $X$ and $\varepsilon>0$ such that $u(C) \not \subset\{y \in X:|\varphi(y)|<\varepsilon\}$. Then the semigroup $S^{*}=\left\{s^{*}\right.$ : $\left.X^{*} \rightarrow X^{*} \mid s \in S\right\}$ of adjoints of $S$ satisfies the assumptions of Theorem 5.8 since it is non-deflating, norm-nonexpansive and $\left(s^{*} \varphi\right)(z)=\varphi(s(z))=\varphi(z)=1$ for every $\varphi \in Q$ and $s \in S$. Hence there exists $\psi \in Q$ such that $\psi(s(x))=\psi(x)$ for all $x \in X$ and $s \in S$. Thus Theorem 5.8 is a nonlinear generalization of [9, Theorem 1] in the case of reflexive spaces.

Acknowledgements The author is grateful to the referee whose valuable comments have improved the presentation of the paper.

Open Access This article is licensed under a Creative Commons Attribution 4.0 International License, which permits use, sharing, adaptation, distribution and reproduction in any medium or format, as long as you give appropriate credit to the original author(s) and the source, provide a link to the Creative Commons licence, and indicate if changes were made. The images or other third party material in this article are included in the article's Creative Commons licence, unless indicated otherwise in a credit line to the material. If material is not included in the article's Creative Commons licence and your intended use is not permitted by statutory regulation or exceeds the permitted use, you will need to obtain permission directly from the copyright holder. To view a copy of this licence, visit http://creativecommons.org/licenses/by/4.0/.

\section{References}

1. Alspach, D.E.: A fixed point free nonexpansive map. Proc. Am. Math. Soc. 82, 423-424 (1981)

2. Bader, U., Gelander, T., Monod, N.: A fixed point theorem for $L_{1}$ spaces. Invent. Math. 189, 143-148 (2012)

3. Bartoszek, W.: Nonexpansive, $\mathcal{T}$-continuous antirepresentations have common fixed points. Proc. Am. Math. Soc. 127, 1051-1055 (1999)

4. Bourgin, R.D.: Geometric aspects of convex sets with the Radon-Nikodým property. Springer-Verlag, Berlin (1983)

5. Boyce, W.M.: Commuting functions with no common fixed point. Trans. Am. Math. Soc. 137, 77-92 (1969)

6. Brodskiĭ, M.S., Mil'man, D.P.: On the center of a convex set. Doklady Akad. Nauk SSSR 59, 837-840 (1948). (in Russian)

7. Bruck Jr., R.E.: A common fixed point theorem for a commuting family of nonexpansive mappings. Pacific J. Math. 53, 59-71 (1974) 
8. DeMarr, R.: Common fixed points for commuting contraction mappings. Pacific J. Math. 13, 1139$1141(1963)$

9. Fan, K.: Orbits of semi-groups of contractions and groups of isometries. Abh. Math. Sem. Univ. Hamburg 45, 245-250 (1976)

10. Furstenberg, H.: The structure of distal flows. Am. J. Math 85, 477-515 (1963)

11. Glasner, E.: Compressibility properties in topological dynamics. Am. J. Math. 97, 148-171 (1975)

12. Glasner, E., Megrelishvili, M.: On fixed point theorems and nonsensitivity. Israel J. Math. 190, 289-305 (2012)

13. Hansel, G., Troallic, J. P.: Démonstration du théor ème de point fixe de Ryll-Nardzewski par extension de la méthode de F. Hahn, C. R. Acad. Sci. Paris Sér. A-B 282 , Aii, A857-A859 (1976)

14. Hsu, R.: Topics on weakly almost periodic functions, Ph.D. Thesis, SUNY at Buffalo, (1985)

15. Huneke, J.P.: On common fixed points of commuting continuous functions on an interval. Trans. Am. Math. Soc. 139, 371-381 (1969)

16. Jayne, J.E., Rogers, C.A.: $K$-analytic sets, in analytic sets, pp. 1-181. Academic Press, London (1980)

17. Lau, A.T., Takahashi, W.: Invariant means and fixed point properties for non-expansive representations of topological semigroups. Topol. Methods Nonlinear Anal. 5, 39-57 (1995)

18. Lau, A.T.-M., Zhang, Y.: Fixed point properties for semigroups of nonlinear mappings and amenability. J. Funct. Anal. 263, 2949-2977 (2012)

19. Losert, V.: The derivation problem for group algebras. Ann. of Math. (2) 168, 221-246 (2008)

20. Megrelishvili, M.: Fragmentability and continuity of semigroup actions. Semigroup Forum 57, 101126 (1998)

21. Namioka, I.: Right topological groups, distal flows and a fixed point theorem. Math. Syst. Theory $\mathbf{6}$, 193-209 (1972)

22. Namioka, I.: Kakutani-type fixed point theorems: a survey. J. Fixed Point Theory Appl. 9, 1-23 (2011)

23. Namioka, I., Phelps, R.R.: Banach spaces which are Asplund spaces. Duke Math. J. 42, 735-750 (1975)

24. Ryll-Nardzewski, C.: Generalized random ergodic theorems and weakly almost periodic functions. Bull. Acad. Polon. Sci. Sér. Sci. Math. Astronom. Phys. 10, 271-275 (1962)

25. Ryll-Nardzewski, C.: On fixed points of semigroups of endomorphisms of linear spaces, Proc. Fifth Berkeley Sympos. Math. Statist. and Probability (Berkeley, Calif., 1965/66), Univ. California Press, Berkeley, Calif., pp. 55-61 (1967)

26. Veech, W.A.: A fixed point theorem-free approach to weak almost periodicity. Trans. Am. Math. Soc. 177, 353-362 (1973)

Publisher's Note Springer Nature remains neutral with regard to jurisdictional claims in published maps and institutional affiliations. 